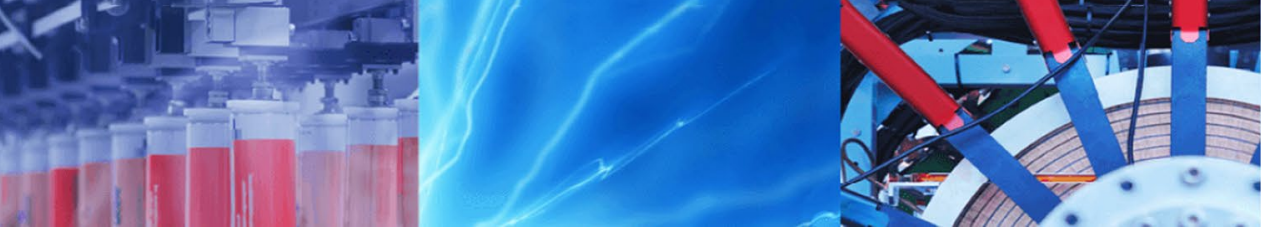

Research Article

\title{
Microstructural characterization and mechanical property prediction of a polymer matrix composite by X-ray synchrotron tomography and spatial correlation functions
}

\author{
Somya Singh ${ }^{1}$. Hechao $\mathrm{Li}^{2}$. Sudhanshu S. Singh ${ }^{1,3}$. Jason Williams ${ }^{1}$ Tyler Stannard ${ }^{1}$ Xianghui Xiao ${ }^{4,5}$. Yang Jiao ${ }^{1}$. \\ Nikhilesh Chawla' ${ }^{1}$
}

๑) Springer Nature Switzerland AG 2019

\begin{abstract}
Polymer matrix composites have become increasingly popular due to their ability of achieving high stiffness and strength without sacrificing desirable ductility. In the present work, we investigate the mechanical properties of borosilicate glass spheres reinforced polypropylene matrix composites with varying volume fractions of the glass spheres, using both tensile tests and nanoindentation measurements. The objective was to study the effect of volume fraction of borosilicate glass spheres on the mechanical behavior of the overall composite system. During the tensile test, the strain in the sample was calculated through three different methods: extensometer measurement, digital image correlation and image processing. The results from these three methods were discussed and compared. X-ray tomography was used for 3D characterization of the microstructure and to investigate failure mechanisms. Additionally, we also developed and validated a systematic procedure for predicting mechanical properties of the composite system by using constituent properties of individual phases coupled with microstructural information obtained from X-ray tomography, quantified via spatial correlation functions. This part of the study focuses on using minimum number of slices to predict mechanical properties, and this is very crucial as handling large 3D datasets is very complicated and time-consuming.
\end{abstract}

Keywords Polymer matrix composites $\cdot$ Mechanical properties $\cdot$ X-ray tomography $\cdot$ Correlation functions

\section{Introduction}

There has been an increasing demand for materials that can demonstrate reliability and desirable performance under extreme conditions. This has led to a shift in interest from monolithic to composite materials in the past few decades. Polymer matrix composites (PMCs) have been tailored to obtain high stiffness, strength-to-weight ratio, fracture toughness and ductility [1-4]. They are being extensively used in structural applications, such as aircraft and automobiles. These materials can be tailored by changing the type and amount of reinforcement to achieve the desired properties. Thus, a thorough understanding of the microstructure and mechanical properties of materials is very important.

Traditionally, the microstructure of a material is studied through two dimensional (2D) characterization techniques, such as optical microscopy and scanning electron microscopy. However, 2D studies are sometimes inaccurate or insufficient as the surface of materials can have distinctively different properties from the bulk. In addition, it is well known that topologically connectedness information of a 3D structure cannot be accurately obtained based on analysis of 2D slices. As a result, three-dimensional (3D)

\footnotetext{
$\triangle$ Nikhilesh Chawla, nchawla@asu.edu | ${ }^{1}$ Materials Science and Engineering, Arizona State University, Tempe, AZ 85287-6106, USA. ${ }^{2}$ Mechanical Engineering, Arizona State University, Tempe, AZ 85287, USA. ${ }^{3}$ Department of Materials Science and Engineering, Indian Institute of Technology Kanpur, Kanpur, Uttar Pradesh 208016, India. ${ }^{4}$ Argonne National Laboratory, Lemont, IL 60439, USA. ${ }^{5}$ Brookhaven National Laboratory, Upton, NY 11973, USA.
} 
characterization techniques are becoming increasingly popular [5]. Among them, X-ray tomography has emerged as one of the most popular characterization techniques as it is nondestructive and precludes the need for extensive sample preparation $[6,7]$. It also allows the user to study the time-dependent behavior of materials under external stimuli in situ, commonly known as 4D experiments. Furthermore, it is capable of achieving high temporal and spatial resolutions at the micrometer or nanometer length scales. These datasets then can also be used to perform microstructure-based modeling to understand deformation behavior. Hence, a number of studies have utilized the capabilities of X-ray tomography to deepen the understanding of microstructure and properties of various material systems [8-13].

In the present work, we have investigated the mechanical properties of a model polymer matrix composite system containing borosilicate glass spheres embedded in a polypropylene matrix. Polypropylene has excellent thermal, mechanical and electrical properties and is one of the most widely used polymers. It can also be easily recycled at the end of its lifetime and has applications ranging from consumer goods to automotive applications, which made it an ideal choice for this work [14-16]. Borosilicate glass spheres were chosen as reinforcement for this model system to get optimum contrast between the matrix and reinforcement in the X-ray images. The effect of the sphere volume fraction on the stiffness and strength of the composite system was studied via both tensile testing and nanoindentation. The strain during tensile testing was obtained through three different methods: use of an extensometer, image-based measurement of the displacement between two points and digital image correlation (DIC). The results from these three methods are discussed and compared. We also developed and validated a systematic procedure for predicting mechanical properties of the composite system (i.e., elastic moduli), without explicit numerical simulations, by employing analytical formalisms derived from effective medium theory (EMT). This is done by extracting key structural information from X-ray tomography data through certain spatial correlation functions and coupling it with individual phase properties obtained from nanoindentation and tensile testing. The major advantage of this procedure is that only a small number of the processed 2D images of the composite microstructure were necessary and sufficient to derive the required microstructural information. This removes the need of extensive image processing of the entire dataset which is a very time-consuming and laborious task.

\section{Materials and experimental procedure}

\subsection{Materials}

Model systems of polymer matrix composites containing borosilicate glass spheres (Bay Materials, CA) embedded in a polypropylene matrix were used in this study. These PMCs contained $5 \%, 10 \%, 20 \%$ and $30 \%$ volume fraction of reinforcement. The borosilicate glass spheres (having a diameter in the range of 38-45 $\mu \mathrm{m}$ ) were mixed with polypropylene using a Brabender mixer, and a different batch for each volume fraction was prepared. This was then used to obtain a sheet of $100 \mathrm{~mm} \times 100 \mathrm{~mm} \times 3.1 \mathrm{~mm}$ each through compression molding. The motivation behind choosing this composite system came from the significant difference in the mass attenuation coefficient between the matrix (polypropylene) and the reinforcement (borosilicate), which leads to enhanced contrast and easier image processing downstream.

\subsection{X-ray tomography}

X-ray synchrotron tomography was performed on the polymer matrix composite samples at the Advanced Photon Source (APS) at Argonne National Laboratory. For this particular experiment, the 2-BM beamline was used. Details of this beamline can be found elsewhere [17-19]. The sample was $5 \mathrm{~mm}$ in height and had a near square cross section with the diagonal length being less than $1 \mathrm{~mm}$. This was obtained by cutting and polishing a small piece from the as-received samples. The $X$-ray beam energy was approximately $24 \mathrm{keV}$. A $2560 \times 2016$ pixel PCO Dimax CMOS camera coupled with a LuAG:Ce scintillator screen, which converts $X$-rays to visible light, was used for capturing images during the scan.

The scan was conducted at angular increments of $0.120^{\circ}$ over a range of $180^{\circ}$, and 1500 projections were collected. With a typical exposure time of $100 \mathrm{~ms}$ per projection, a pixel size of $0.65 \mu \mathrm{m}$ was achieved. To understand the effect of phase contrast on the quality of obtained images and further segmentation, the samples were scanned at two sample-to-detector distances, i.e., $25 \mathrm{~mm}$ and $60 \mathrm{~mm}$. TomoPy, an open-source Python toolbox [20], was used for generating the 3D reconstruction from the acquired $2 \mathrm{D}$ projections. Before reconstruction, the obtained 2D projections were processed through 3 steps. The first step normalizes the images by using a smoothing filter on the raw projections. This was followed by stripe removal, which essentially removes vertical artifacts from the sinogram using a Fourier-wavelet 
approach [21]. These stripes, when not removed, were seen as rings in the reconstruction and later interfered with the process of segmentation. Subsequently, phase retrieval was used to enhance the contrast at the boundary of constituent phases [22]. This is achieved by removing stripes from the sinogram of the projections using Titarenko's approach [23]. After processing the 2D projections, 3D reconstruction was performed using the filtered back-projection algorithm [24].

\subsection{Nanoindentation}

Nanoindentation was performed to obtain the Young's modulus of the borosilicate glass spheres and the polymer matrix using a commercial nanoindenter (Nanoindenter XP-II, Agilent). The specimens were polished to a final finish of $0.05 \mu \mathrm{m}$ by colloidal silica. Indentations were performed using a Berkovich tip to a depth of $1000 \mathrm{~nm}$. Tests were strain-rate-controlled with a strain rate target of $0.05 \mathrm{~s}^{-1}$. Before indenting the specimens, the calibration of nanoindenter was done by measuring Young's modulus and hardness of a standard silica sample.

Nanoindentation was carried out on at least 20 different borosilicate particles and at 35 different locations in the matrix. Continuous stiffness measurement (CSM) technique was used during nanoindentation $[25,26]$ instead of the conventional Oliver and Pharr's method. In the CSM method, a small amplitude of oscillating force is superimposed on the load that is applied onto the specimen surface. Further, by separating the in-phase and out-of-phase components of the load-displacement data, the contact stiffness is obtained for indentation depths. The contact stiffness is further used for calculating Young's modulus and hardness [25]. The advantage of CSM technique is that it enables instantaneous measurements of modulus and hardness during loading, thus avoiding the need for conducting multiple indentations at different depths. Young's modulus and hardness for an individual indentation were measured as the average value over a depth range where both modulus and hardness were independent of depth.

\subsection{Tensile testing, digital image correlation (DIC) and image-based measurement of strain}

Mechanical properties of the composite systems, as well as the unreinforced polypropylene, were obtained by testing dog-bone and rectangular samples in a MTS 810 servo hydraulic testing machine. Tensile tests were carried out at a strain rate of $10^{-3} \mathrm{~s}^{-1}$. The dog-bone samples were made in accordance with ASTM D638 having a gage length of $7.6 \mathrm{~mm}$ and a total length of $63.5 \mathrm{~mm}$.

Photogrammetry or digital image correlation method has been successfully used for local strain field measurements in metallic materials with heterogeneous microstructure as well as for stress concentration studies in structural materials containing notches, holes, etc. [27-32]. On loading the sample, its surface gets stretched and hence any pattern that might be present on the surface naturally or that was made artificially (speckle pattern) changes its position [27,33]. Digital image correlation method is based on the tracking of geometrical changes in the grayscale distribution of these patterns during the test for strain field measurement.

A speckle pattern was applied on the sample. The speckle pattern was made on the sample with the help of an airbrush (Iwata-Eclipse series). The $0.35-\mathrm{mm}$ needle-nozzle combination of the brush helped in fine detailed spraying. For better adherence between the sample and paint, the surface of the sample was cleaned using acetone. First, a base of white paint was made and allowed to dry for $20 \mathrm{~min}$, after which the pattern was made using black color water-based paint as shown in Fig. 1a. It was ensured that the entire surface was patterned and a good adherence between the sample and paint was achieved.

Strain values were measured using digital image correlation (DIC) and image processing through thresholding. Further, rectangular specimens having dimensions of $(100 \mathrm{~mm} \times 10 \mathrm{~mm} \times 3 \mathrm{~mm}$ ) were machined and strain was measured using an extensometer. These three techniques

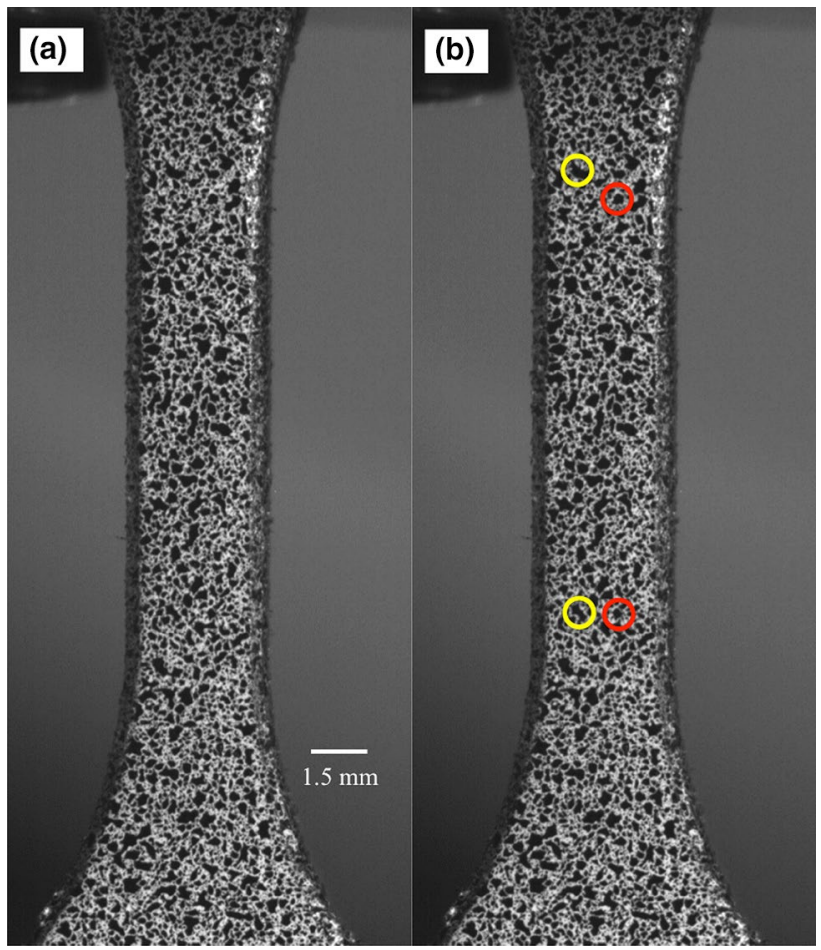

Fig. 1 a Speckle pattern on the sample using water-based white and black paints to measure strains using DIC, b 2 pairs of features selected for calculation of strain 
were used to obtain statistically relevant results and validate the accuracy of each method.

To record the deformation during the tensile tests, a camera (PixeLink Industrial Cameras, Canada) was adjusted perpendicular to the surface of the sample and images were taken every $3 \mathrm{~s}$. The camera was equipped with a lens of $50 \mathrm{~mm}$ focal length (Schneider-Kreuznach, Germany). These grayscale images were then used to calculate the displacement field and the associated strain using DIC through ARAMIS software. In ARAMIS, a grid of square facets is mapped on the first acquired image of the unstrained sample. The dimension of the square and the distance between them are important and are known as facet size and step size, respectively [27]. These values are manually entered and can be adjusted in accordance with the type of speckle pattern. This is crucial, as each facet must contain enough pixels to obtain an adequate grayscale distribution.

In the unstrained condition, each facet is characterized by the grayscale distribution within the square and the coordinates of the facet centers [34]. During straining, the grayscale pattern gets modified and is tracked based on the underlying assumption that this distribution remains constant around a certain coordinate. Through the tracking of these changes in the grayscale distribution, displacement gradient tensor field is determined at each facet center. This then helps in obtaining the strain tensor through the surface components of local displacement gradient tensor. Calibration was performed by correlating two undeformed images. The estimated residual error was $0.027 \%$, which is well below the accepted level of $0.04 \%$ [27]. For the present work, all the images that were acquired during the test at a regular interval of $3 \mathrm{~s}$ were imported into the software and each image corresponded to a particular stage. After this, a region of interest (within the gauge section) for the grid of facets was determined. The values of facet size and the step size were $550 \mu \mathrm{m}$ and $233 \mu \mathrm{m}$, respectively. Through DIC, local and average values of strain for the entire gage length were obtained.

The average strain was also measured by measuring the relative displacement of images from two areas in the sample. The grayscale images were imported into the Image J software and binarized using conventional thresholding. To measure the strain, a set of features, circular in shape, was selected in the gauge section, as shown in Fig. 1b. The distance between their centroids during the test was used to calculate the strain. It should be mentioned here that on being strained, the change in shape of the pattern itself can lead to change in the value of centroid. Therefore, two such pairs of features were selected for measurements of strains and the final value was taken as the average of the two strains. In comparison with the DIC, where a map of strain distribution on the surface is obtained, strain values obtained from the thresholding method are the average value through the gage section.

A conventional extensometer was also used to measure strains on the rectangular sample $100 \mathrm{~mm} \times 10 \mathrm{~mm} \times 3 \mathrm{~mm}$. The test was to be conducted only in the elastic regime, so a rectangular geometry was used. The test was conducted at a strain rate of $10^{-3} \mathrm{~s}^{-1}$. In order to obtain statistically relevant results, the tests were done four times for each volume fraction of composite and for the unreinforced sample. For each sample, extensometer was placed on all four faces of the sample and average value from those measurements was used to obtain Young's modulus. These values were further compared with the results obtained from DIC and image processing.

\section{Results and discussion}

\subsection{Image segmentation}

X-ray synchrotron tomography can be conducted using absorption contrast and phase contrast. The proportion of absorption and phase contrast depends upon the distance between the sample and detector. As the distance between the sample and detector increases, the proportion of phase contrast (refraction) increases. Figure $2 a, c$ shows the $2 \mathrm{D}$ reconstructed slices of PMC with $30 \%$ volume fraction of borosilicate particles scanned at sampleto-detector distances of $25 \mathrm{~mm}$ and $60 \mathrm{~mm}$, respectively. It can be seen that the proportion of phase contrast, which is visible in the form of the dark periphery around the borosilicate particles, is higher in the case of $60 \mathrm{~mm}$ compared to $25 \mathrm{~mm}$. These are called near-field phase contrast fringes (mainly first-order interference fringes), which form due to the interference between the incident and refracted waves in phase contrast imaging. Moreover, ring artifacts were also more prominent in the images obtained at a sample-to-detector distance of $60 \mathrm{~mm}$ than $25 \mathrm{~mm}$. The increased amount of both, near-field phase contrast fringes and ring artifacts, led to the problems in the segmentation. Therefore, datasets obtained from scanning at a sample-to-detector distance of $25 \mathrm{~mm}$ were used for image processing and analysis.

Figure 3 shows reconstructed 2D images of the composites with $5 \%, 10 \%, 20 \%$ and $30 \%$ volume fraction of reinforcement. For visualization and quantification of the $3 \mathrm{D}$ microstructure, these grayscale datasets were segmented. Several steps were performed in the segmentation process for better accuracy and efficiency. The dataset was first processed by anisotropic diffusion filter, which is also known as the Perona-Malik diffusion filter [35, 36]. It aims at reducing the noise in images without removing important features such as edges and cracks. These 

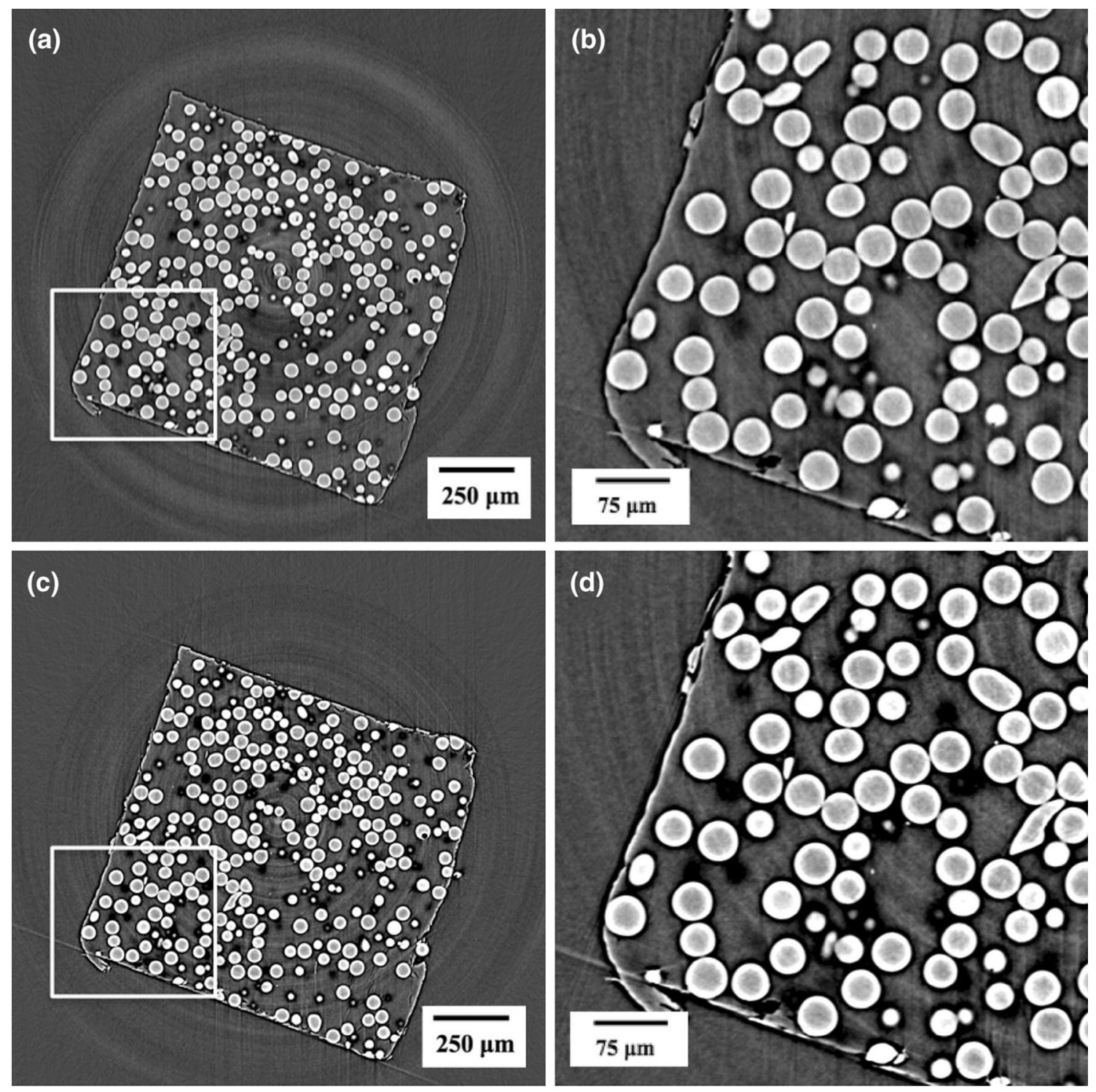

Fig. 2 A single slice from the reconstructed dataset of scan performed at a $25 \mathrm{~mm}$ distance and c $60 \mathrm{~mm}$ distance between sample and detector. With the increase in sample-to-detector distance,

grayscale images were then binarized through thresholding in ImageJ software, which is based on an ISODATA algorithm [37]. However, due to the presence of ring artifacts in the reconstructed image, unwanted particles were present in the images after thresholding, as shown in Fig. 4b (marked by arrow). Some of these were removed through pixel erosion followed by a watershed filter [38] to separate the particles that were erroneously touching. The resultant image is shown in Fig. 4c.

Finally, "fill holes" and "analyze particles" features of ImageJ were used to fill the inside of incompletely filled particles and to remove the small undesired particles remaining from the ring artifacts, respectively. In phase contrast increases that is seen in the form of dark outline of borosilicate glass spheres. This can be seen from the magnified area shown in (b) and (d)

"analyze particles," the minimum and maximum pixel area sizes were specified and the particles outside this size window were removed. Figure $4 d$ shows the final segmented image. These segmented datasets were then used for visualization of the 3D microstructure along with the prediction of mechanical properties through microstructure-based models. Figure 5 shows the 3D rendering of the composite systems generated through Avizo ${ }^{\circ}$ a commercially available software.

An attempt was made in this study to minimize the amount of data required and through strong-contrast expansion formulism, and the mechanical properties 

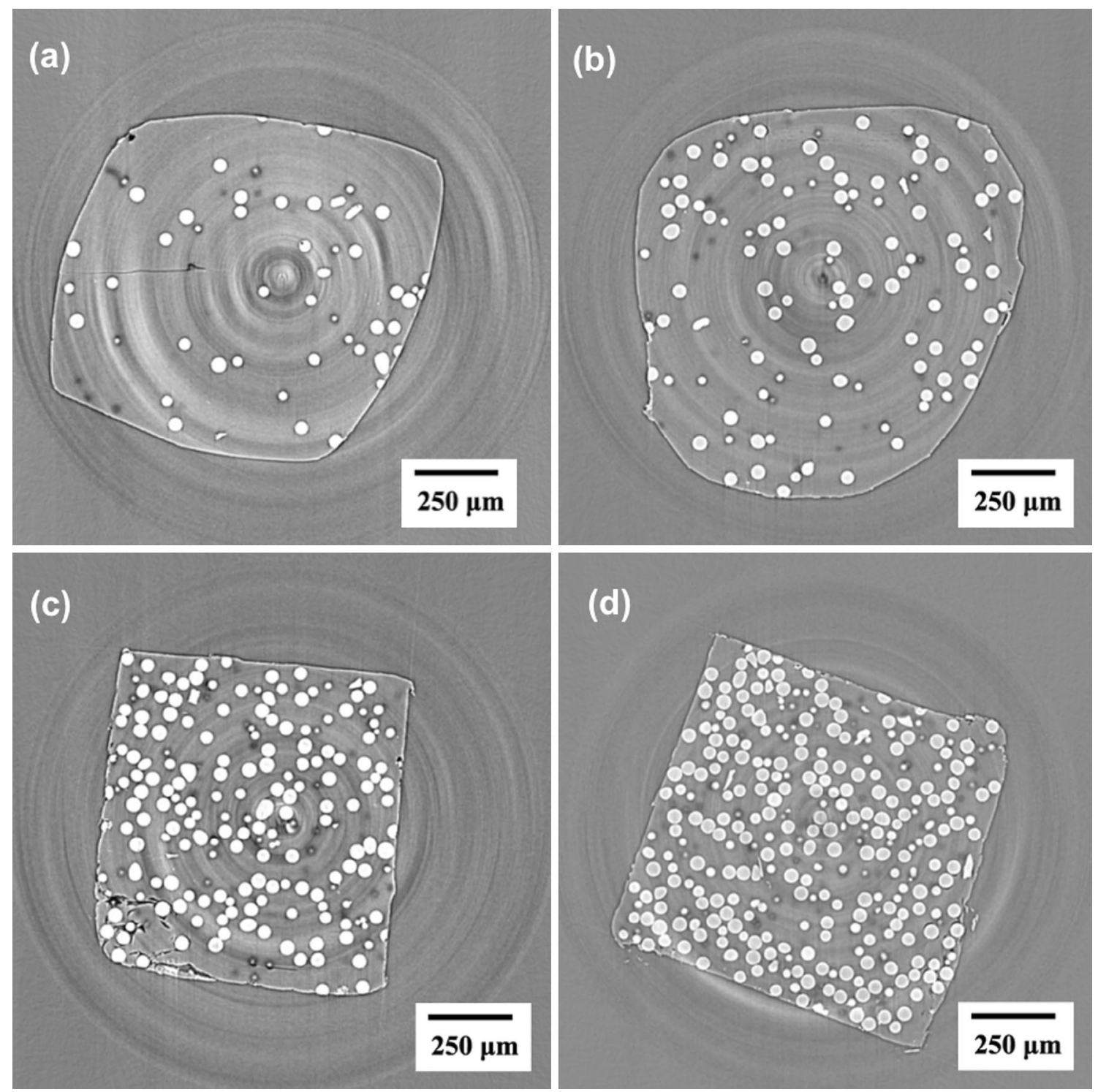

Fig. 3 A single slice from the reconstructed dataset of a PMC 5\%, b PMC 10\%, c PMC 20\% and d PMC 30\%

were predicted using microstructural information that was derived from a couple of segmented slices.

\subsection{Nanoindentation}

Young's modulus curves as a function of depth for the matrix and particle are shown in Fig. $6 a$ and b, respectively. Figure 7 shows the SEM indent of image on particle. For borosilicate glass spheres, results between a depth of 60 and $150 \mathrm{~nm}$ were used for the calculations and a modulus of $46.1 \mathrm{GPa}$ was obtained. The polypropylene matrix is much more compliant than the borosilicate particles which led to a gradual decrease in the particle modulus at greater depths. This can be attributed to substrate effect due to the presence of an underlying softer matrix. Thus, the modulus was calculated over the range of displacement where the modulus was independent of depth and the effect of the underlying matrix was minimal. The Young's modulus values of the matrix remained constant from 300 to $800 \mathrm{~nm}$, and an average value of $2.0 \mathrm{GPa}$ was obtained. The Young's modulus of borosilicate glass spheres was then used as inputs for predicting the Young's bulk modulus and shear modulus of composites containing different volume fraction of reinforcements through strong-contrast expansion formulism.

\subsection{Tensile testing}

Tensile tests were done for the unreinforced polypropylene and composite material containing 5\%, 10\%, 20\% and 


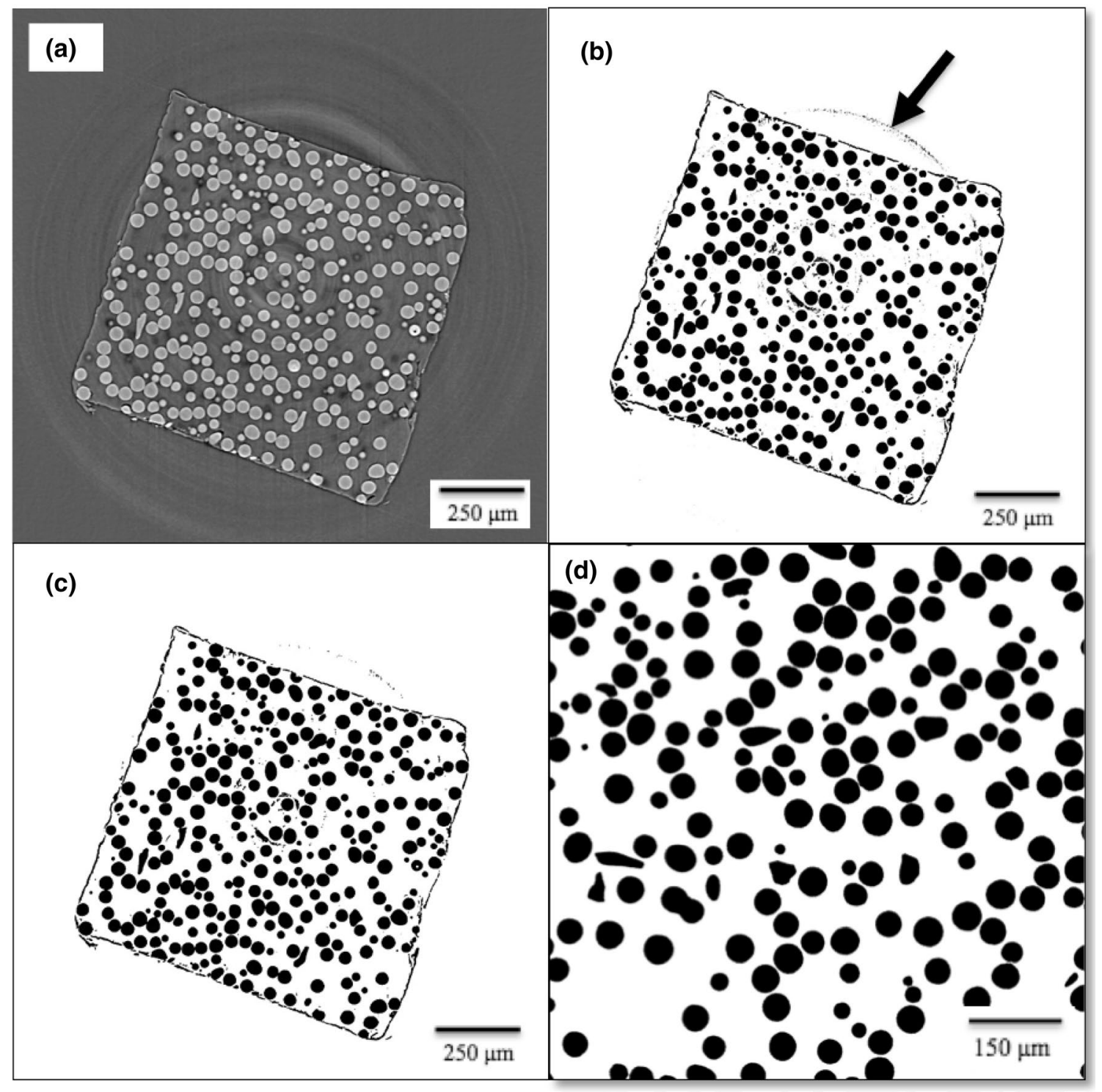

Fig. 4 Steps followed for segmenting the dataset a Image after anisotropic diffusion, $\mathbf{b}$ grayscale image converted into 8 bits by thresholding in ImageJ, c "Watershed" was used to disconnect the particles which were erroneously touching, d "Analyze particles" was used to remove the unwanted features and manual correction was done on comparing the segmented slices with initial reconstruction
$30 \%$ volume fraction of reinforcements. Rectangular and dog-bone-shaped samples were tested for these composites. Figure 8 shows the sample with speckle pattern and the strain distribution on the surface of sample obtained from DIC at four different stages during the test. DIC helps in obtaining the strain values throughout the sample surface, and it can be observed that the region where necking initiated underwent more deformation as compared to other regions.

Figure 9 shows the comparison of the Young's modulus obtained from DIC, image processing and extensometer. It can be seen that the results from all three methods are in good agreement with each other. This validates the results from image processing where strain was obtained from tracking the changes in position of centroid of two points within the gage length. It can be seen that the value of Young's modulus increased from 1.1 GPa of unreinforced polypropylene to $2.3 \mathrm{GPa}$ of composite with $30 \%$ volume fraction of reinforcement. This was due to the increase in the volume of stiffer borosilicate glass spheres which enhances the load-bearing capacity of composite system. 
Fig. 5 3D visualization of segmented microstructure of a PMC 5\%, b PMC $10 \%$, c PMC $20 \%$ and d PMC $30 \%$ generated in Avizo (a)

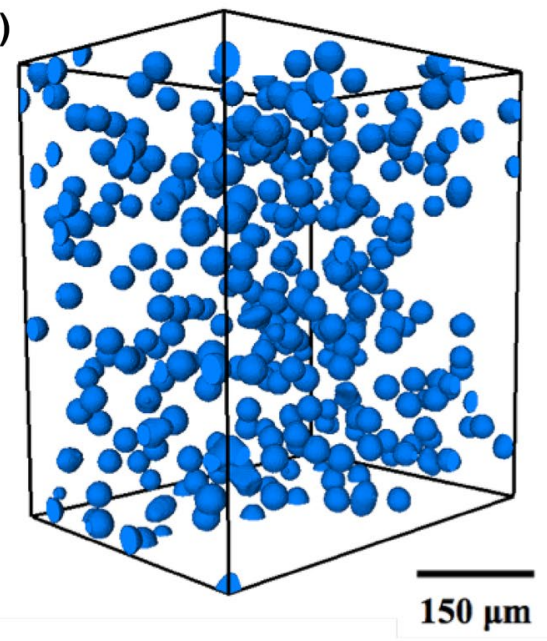

(c)

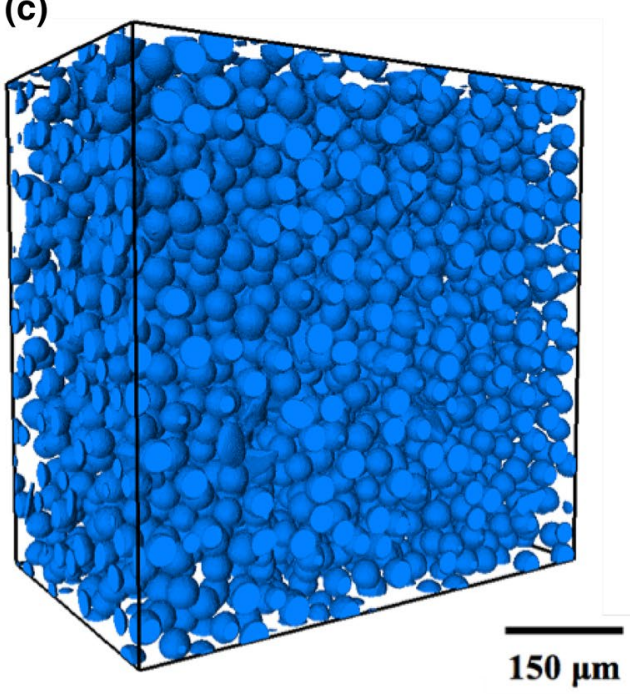

(b)

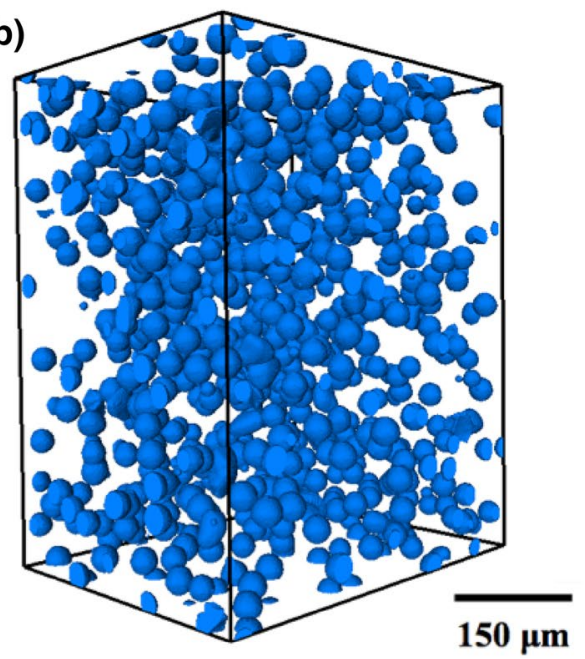

(d)

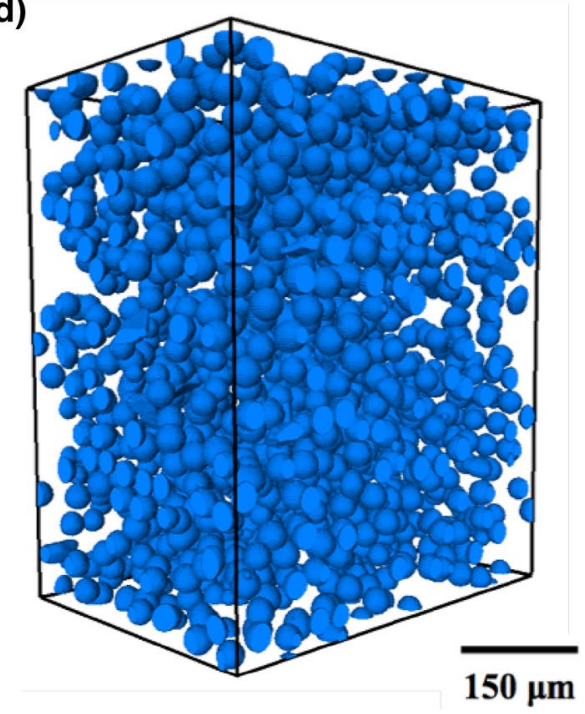

\subsection{Property prediction via spatial correlation functions}

The high-quality imaging data allow us to extract key statistical morphological information of the composite materials, in forms of the n-point correlation function $S_{n}$, for direct mechanical property prediction. This is done by employing the strong-contrast expansion (SCE) formalism that analytically expresses the elastic moduli of a heterogeneous material as a series of integrals involving $S_{n}$ and individual phase properties [39]. The $n$-point correlation function $S_{n}$ generally provides the probability of finding a specific n-point configuration, when randomly placed in the material, with all of the $n$ points falling into the phase of interest [40].

Here, we apply the SCE formalism to estimate the elastic moduli of the composites for different sphere volume fractions and employ the truncated expansion at the third order (involving $S_{3}$ ) for both the bulk modulus $K$ and shear modulus $G$ of the system. $\Phi_{1}$ and $\Phi_{2}$ are, respectively, the volume fractions of the polymer matrix (phase 1 ) and the glass spheres (i.e., phase 2 ), and $d=3$ is the dimension of the system.

$\Phi_{2} \frac{\kappa_{21}}{\kappa_{e 1}}=1-\frac{(d+2)(d-1) G_{1} \kappa_{21} \mu_{21}}{d\left(\kappa_{1}+2 G_{1}\right)} \Phi_{1} \zeta_{2}$

$$
\begin{aligned}
\Phi_{2} \frac{\mu_{21}}{\mu_{e 1}}= & 1-\frac{2 G_{1} \kappa_{21} \mu_{21}}{d\left(K_{1}+2 G_{1}\right)} \Phi_{1} \zeta_{2}-\frac{\left(d^{2}-4\right) G_{1}\left(2 K_{1}+3 G_{1}\right) \mu_{21}^{2}}{2 d\left(K_{1}+2 G_{1}\right)^{2}} \\
& \Phi_{1} \zeta_{2}-\frac{1}{2 d}\left[\frac{d K_{1}+(d-2) G_{1}}{K_{1}+2 G_{1}}\right]^{2} \mu_{21}^{2} \Phi_{1} \eta_{2}
\end{aligned}
$$

The scalar parameters $\kappa_{21}$ and $\mu_{21}$ are, respectively, the bulk modulus polarizability and shear modulus polarizability, i.e., 


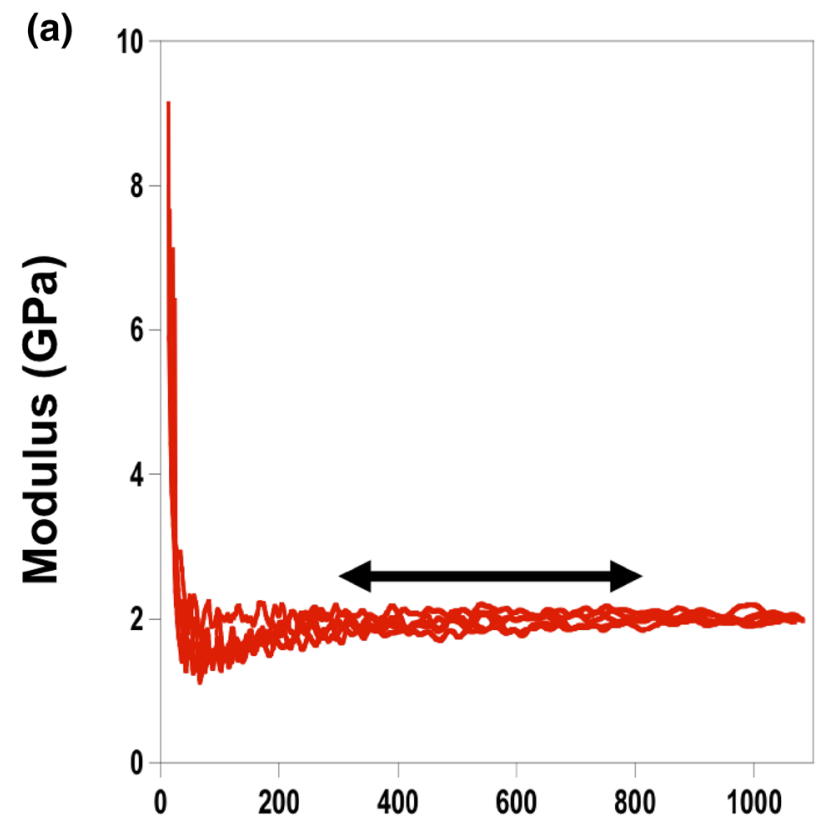

Displacement into the surface $(\mathrm{nm})$

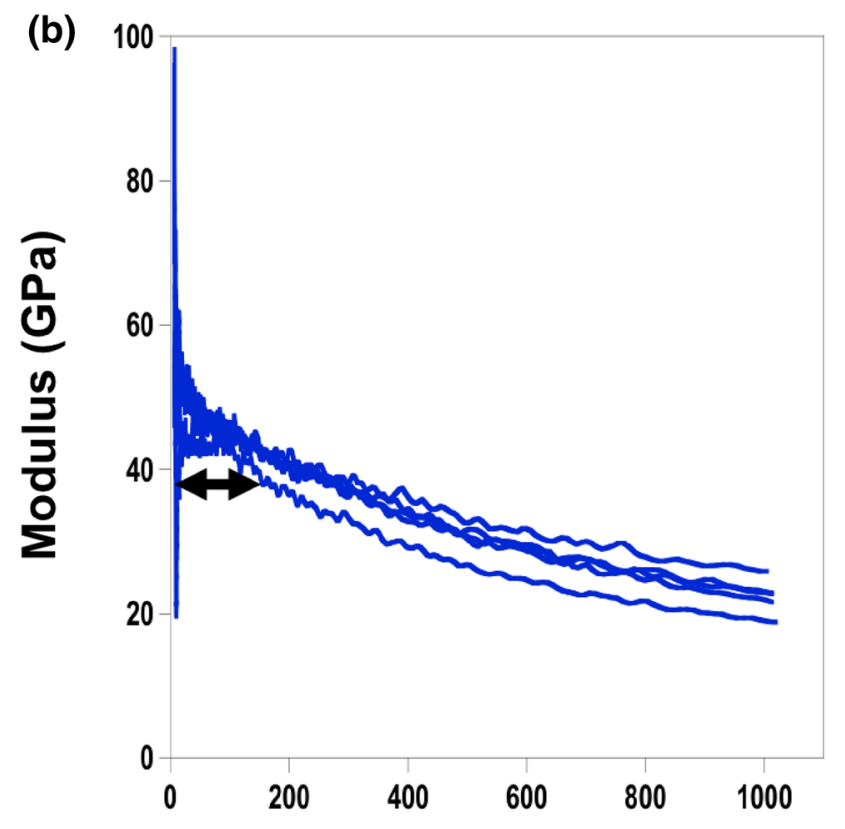

Displacement into the surface $(\mathrm{nm})$

Fig. 6 Young's modulus obtained from nanoindentation curves of a polypropylene matrix, $\mathbf{b}$ borosilicate glass sphere

$\kappa_{21}=\frac{K_{2}-K_{1}}{K_{1}+\frac{2(d-1)}{d} G_{1}}$

$$
\mu_{21}=\frac{G_{2}-G_{1}}{G_{1}+\frac{G_{1}\left[\frac{d K_{1}}{2}+(d+1)(d-2) G_{1} / d\right]}{K_{1}+2 G_{1}}}
$$

$\kappa_{e 1}$ and $\mu_{e 1}$ are the corresponding effective polarizabilities, from which the overall bulk modulus and shear modulus of the composite will be obtained. $K_{1}, G_{1}, K_{2}$ and $G_{2}$ are, respectively, the bulk modulus and shear modulus of the polymer matrix and glass spheres. $\zeta_{2}$ and $\eta_{2}$ are the threepoint parameters associated with the "sphere phase," i.e.,

$$
\begin{aligned}
\zeta_{2}= & \frac{9}{2 \Phi_{1} \Phi_{2}} \int_{0}^{\infty} \frac{\mathrm{d} r}{r} \int_{0}^{\infty} \frac{\mathrm{d} s}{s} \int_{-1}^{1} \mathrm{~d}(\cos \theta) P_{2}(\cos \theta) \\
& {\left[S_{3}^{(2)}(r, s, t)-\frac{S_{2}^{(2)}(r) S_{2}^{(2)}(s)}{\Phi_{2}}\right] } \\
\eta_{2}= & \frac{5 \zeta_{2}}{21}+\frac{150}{7 \Phi_{1} \Phi_{2}} \int_{0}^{\infty} \frac{\mathrm{d} r}{r} \int_{0}^{\infty} \frac{\mathrm{d} s}{s} \int_{-1}^{1} \mathrm{~d}(\cos \theta) P_{4}(\cos \theta) \\
& {\left[S_{3}^{(2)}(r, s, t)-\frac{S_{2}^{(2)}(r) S_{2}^{(2)}(s)}{\Phi_{2}}\right] }
\end{aligned}
$$

where $t=\left(r^{2}+s^{2}-2 r s \cos \theta\right)^{1 / 2}$ and $P_{2}$ and $P_{4}$ are, respectively, the Legendre polynomials of orders two and four,

$P_{2}(x)=\frac{1}{2}\left(3 x^{2}-1\right)$

$P_{4}(x)=\frac{1}{8}\left(35 x^{4}-30 x^{2}+3\right)$

Application of Eqs. (1) and (2) requires the evaluation of the three-point parameters $\zeta_{2}$ and $\eta_{2}$, which involve the three-point correlation function $S_{3}$. To achieve high accuracy of the computed $\zeta_{2}$ and $\eta_{2}$ values, we employ a non-uniform radial sampling template introduced in [41], in which the spatial density of the sampling points monotonically increases (e.g., $\sim 1 / r$ ) as one moves toward the origin along the radial direction. This will provide a sufficient number of sampling points for small $r$ values for an accurate numerical integration. In addition, the mechanical properties (i.e., bulk and shear moduli) of the individual phases required in Eqs. (1) and (2) have been obtained using nanoindentation and tensile test measurements, whereas the overall Young's modulus $E$ of the composites was obtained via tensile testing. The predicted $E$ values for different sphere volume fractions are shown in Figs. 10 and 11 , which agree very well with the corresponding $E$ values obtained from tensile test. 

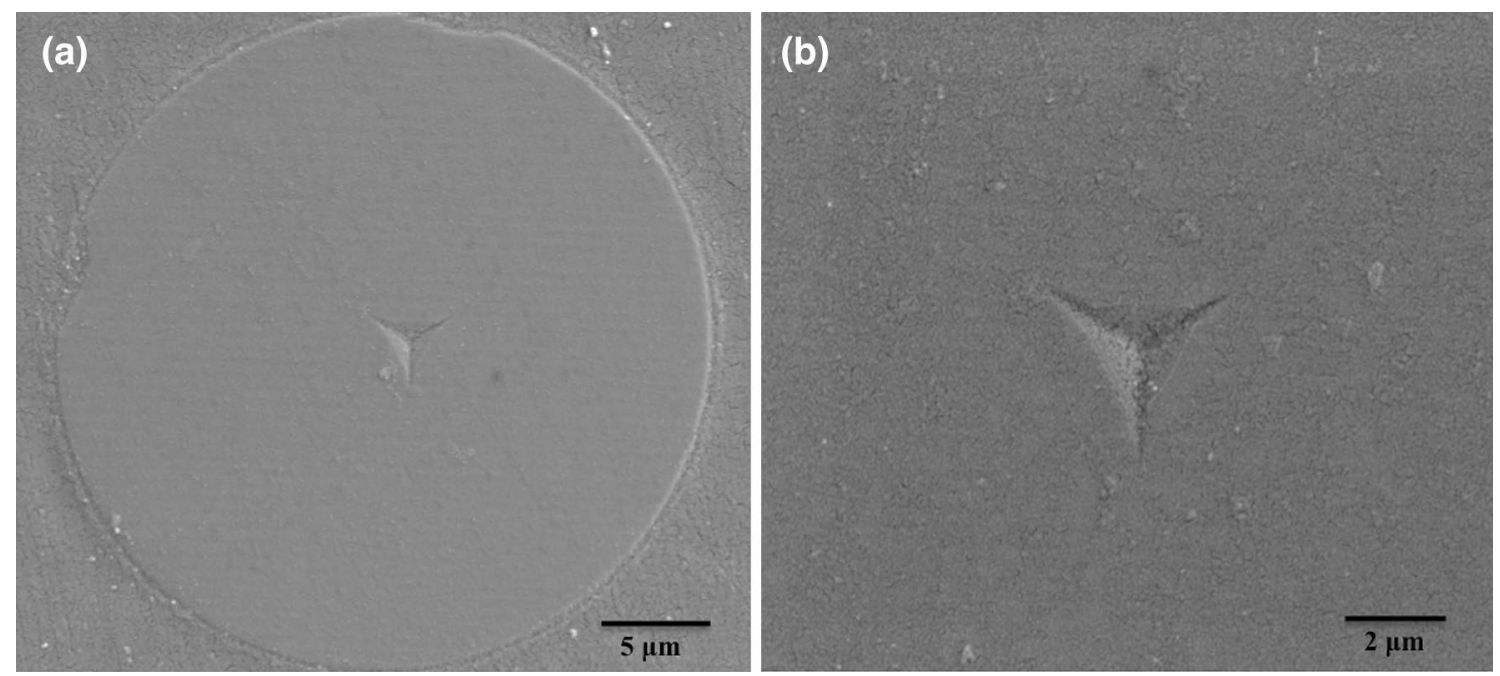

Fig. 7 a SEM image of nanoindentation on borosilicate glass particle. b Magnified image

(a)

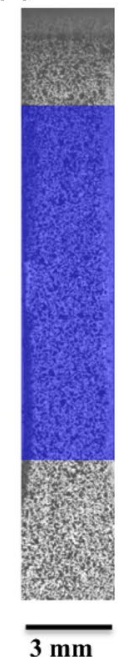

(b)

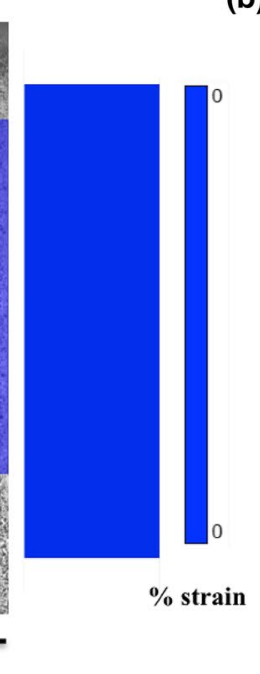

(b)

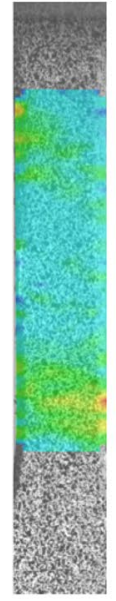

(c)

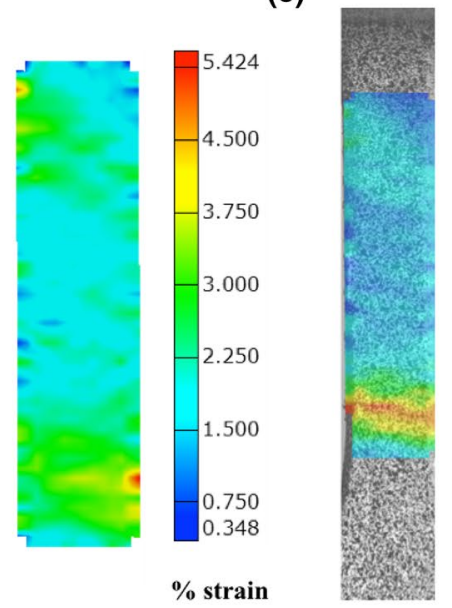

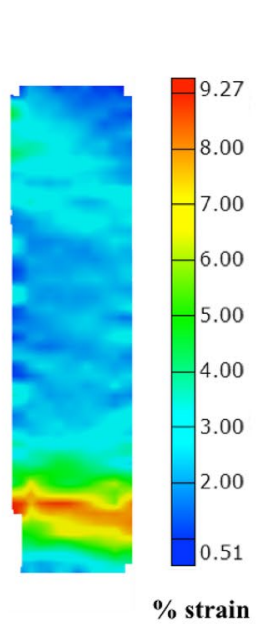

(d)

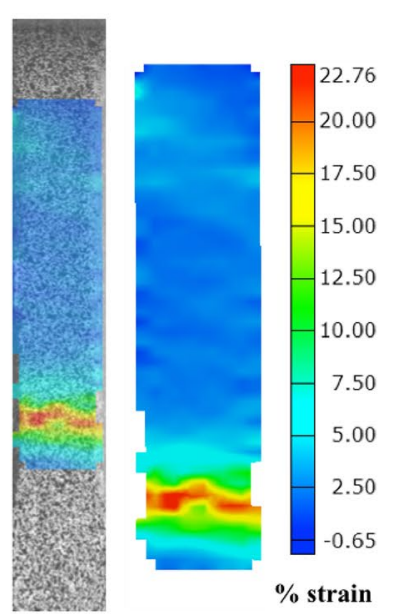

Fig. 8 Highlighted gage length where strain measurements through DIC was taken and localized distribution of strain on the sample for four stages during the test having an average value of strain a $0 \%, \mathbf{b} 0.85 \%, \mathbf{c} 3 \%$, d 4

\section{Validation of property prediction}

\subsection{Young's modulus}

The values of Young's modulus predicted for the composite system through strong-contrast expansion formulism were compared with those obtained from tensile testing. As can be seen from Fig. 10, both the results are in very good agreement with each other. The difference in the predicted and actual results was as low as $1.4 \%$ for composite with $30 \%$ volume fraction of reinforcement. This difference increased on moving to lower volume fraction of reinforcement and was $11.3 \%$ for composite having $5 \%$ volume fraction of borosilicate glass spheres. In this case, though a prediction of $1.1 \mathrm{GPa}$ is very close to the experimentally obtained value of $1.24 \mathrm{GPa}$, the difference can be attributed to the presence of very few particles on one slice of the segmented dataset, which was inadequate to represent the microstructure. This became more clear when the same comparison was done with microstructural information derived from two segmented slices as shown in Fig. 11. Here, the difference in the results for $5 \%$ composite system reduced to less than $1 \%$. Since this was a simple model system, microstructural information derived from a couple of 
(a)

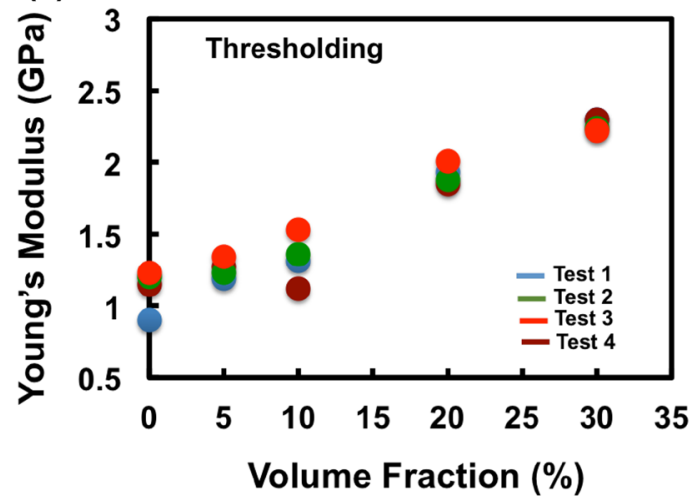

(c)

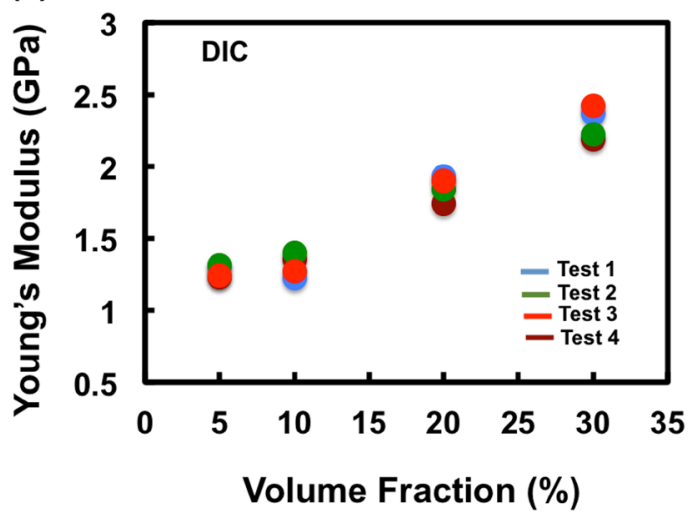

Fig. 9 Young's modulus of unreinforced polypropylene and composite calculated from the stress-strain curves obtained from a image processing via thresholding, b extensometer, $\mathbf{c}$ DIC, $\mathbf{d}$ aver-

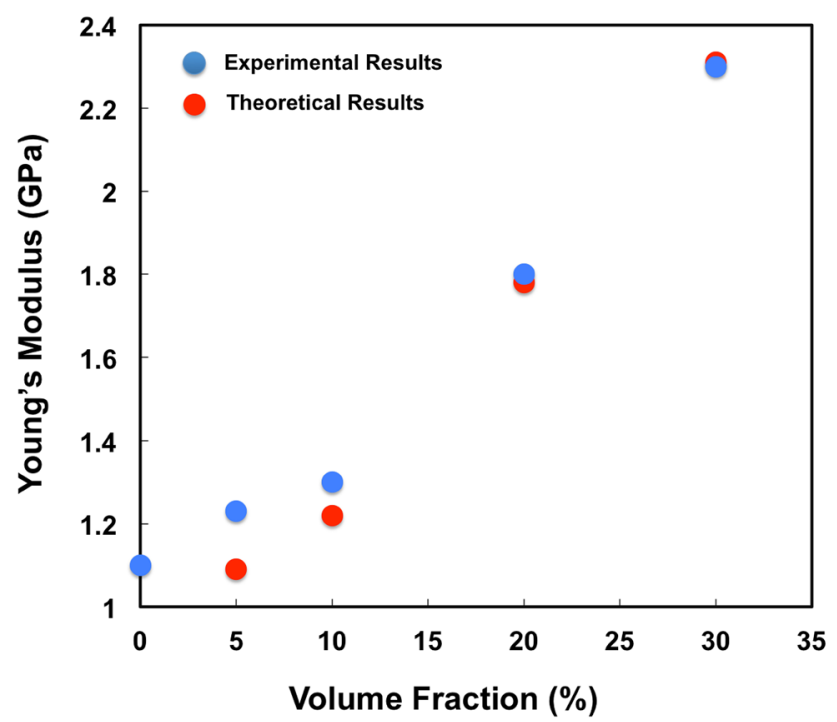

Fig. 10 Comparison of the values of Young's modulus predicted through strong-contrast expansion formalism using one segmented slice with experimental results. The predicted results matched well with experimental results (b)

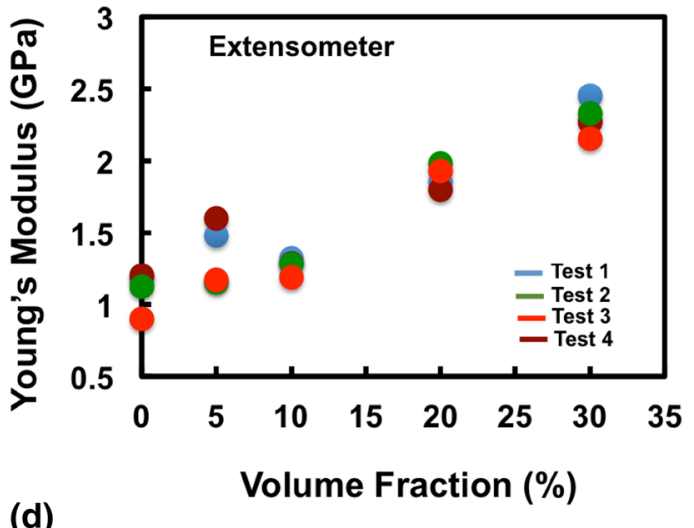

(d)

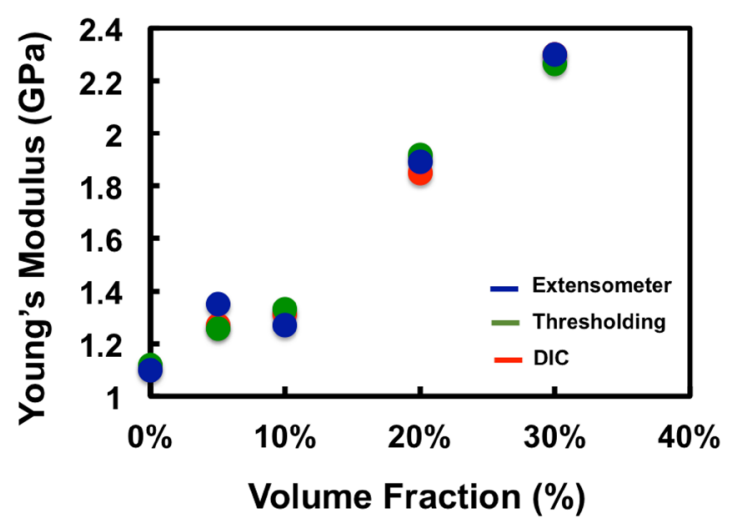

age values from the three methods were compared and are in good agreement with each other

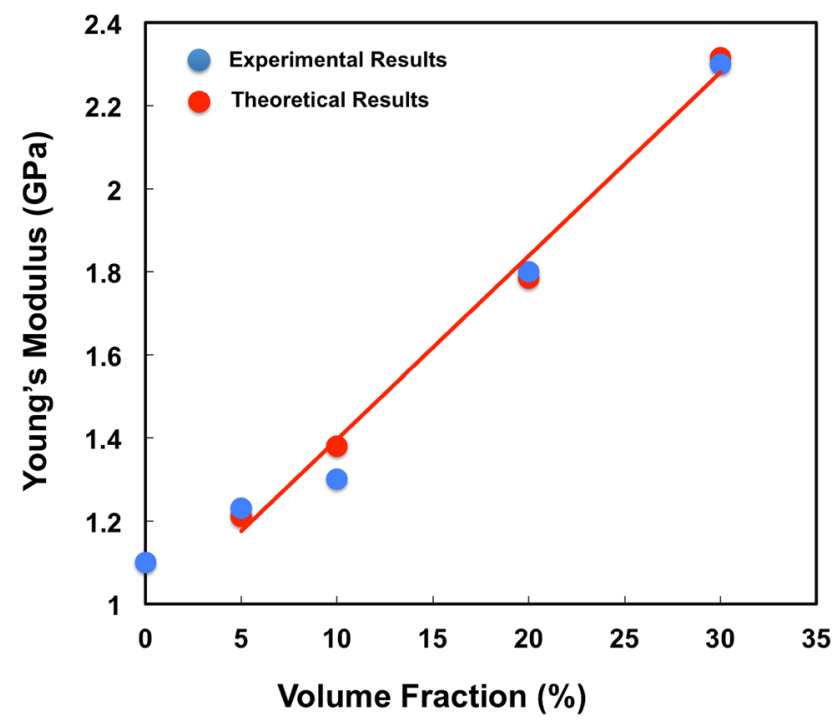

Fig. 11 Comparison of the values of Young's modulus predicted through strong-contrast expansion formalism using two segmented slices with experimental results. An improvement in the predicted result can be observed for $5 \%$ volume fraction, which contains very less number of spheres 
slices was adequate to predict the mechanical properties accurately. This was possible because of the isotropic nature of spherical borosilicate glass spheres, and a few 2D slices in this case are sufficient for its 3D representation. However, with an increase in complexity of microstructures, a couple of segmented slices might not be sufficient for this study and more data might be required for predicting the mechanical properties of the heterogeneous materials accurately.

\subsection{Bulk modulus and shear modulus}

The results of bulk modulus and shear modulus of the composite were compared with the Hashin-Shtrikman bounds. These are well-established bounds that are used to predict the range of composite bulk modulus and shear modulus for two phase materials [42]. On specifying the volume fraction of the reinforcement, upper and lower bounds for the composite are calculated by using equation. For calculating these bounds, the moduli of individual components are interchanged with their respective volume fraction. If one phase has zero elastic moduli, the lower bound becomes zero and thus only the upper bound is useful. Bulk modulus and shear modulus of the composite system should lie within these bounds and can change value depending on the nature of microstructure. The Hashin-Shtrikman bounds for the composite system were calculated by giving the individual modulus and volume fractions as input. The results obtained in Sect. 3.4 were then compared with these bounds. It was seen from Fig. 12 that the obtained values coincided with the lower bound. This was due to the spherical nature of the reinforcement which gives it a low aspect ratio and hence a better fit with the lower bound.

\section{Conclusions}

The microstructure and mechanical properties of the PMC model system containing borosilicate glass spheres embedded in polypropylene matrix were studied. 3D $\mathrm{X}$-ray tomography helped in capturing the microstructure, whereas nanoindentation and tensile tests were performed for obtaining the Young's modulus. The effect of particle volume fraction on the stiffness and strength of the composite was evaluated. Moreover, a systematic procedure was developed for predicting mechanical properties of the composite system using analytical formalisms. Both the data from X-ray tomography and individual phase properties from nanoindentation as well as tensile testing were used. It can be concluded that:

- From the experimental investigation, it was established that there was an increase in the Young's bulk modulus and shear modulus of the composite with increase in volume fraction of stiffer reinforcement. The Young's modulus increased from 1.1 to $2.3 \mathrm{GPa}$ with an increase in borosilicate glass spheres reinforcement from 0 to $30 \%$.

- The strain in the sample was calculated through three different methods: strain from extensometer, DIC and image processing. Extensometer provided the overall (a)

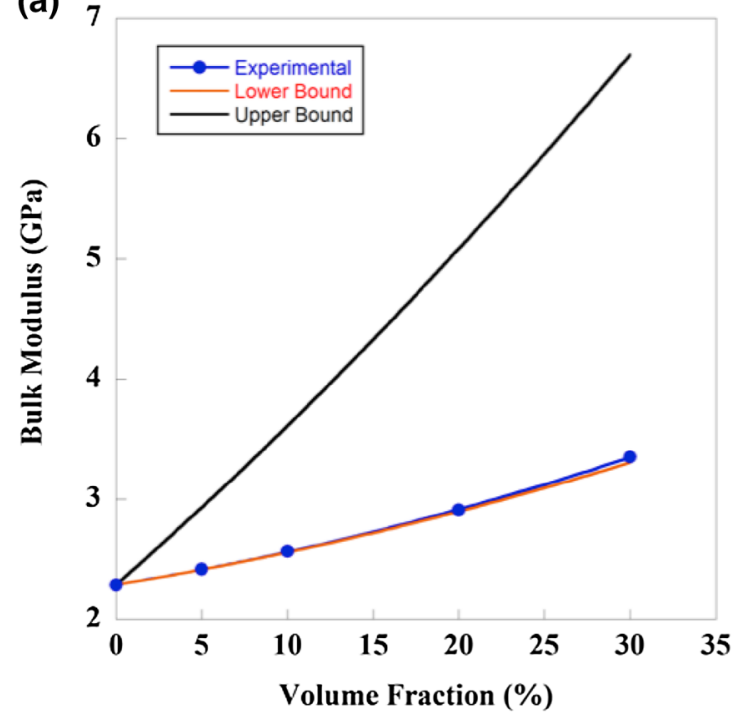

(b)

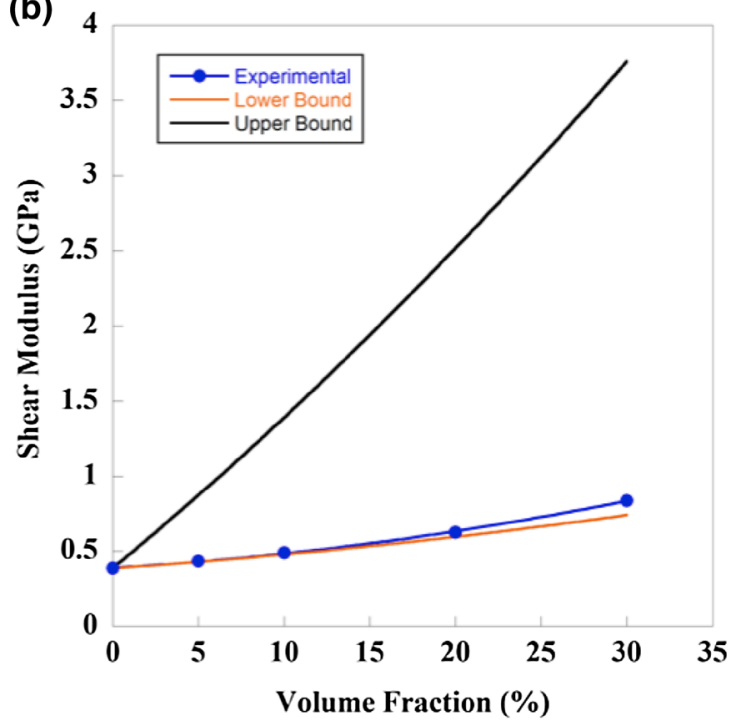

Fig. 12 Results of a bulk modulus and $\mathbf{b}$ shear modulus compared with Hashin-Shtrikman bounds. The results coincided with the lower bounds due to low aspect ratio of spherical borosilicate reinforcement particles 
strain of the sample, whereas DIC helped in mapping strain value on the sample surface. It was seen that the results from these three methods were in good agreement with each other.

- Microstructural information derived from 3D X-ray tomography through three-point correlation function along with the constituent phase properties was used to predict the mechanical properties of the composite through strong-contrast expansion formulism. An excellent match was seen between the predicted and experimental values even though segmentation of just two slices was used for deriving the three-point parameters.

Acknowledgements This work was supported by the Division of Materials Research at the National Science Foundation under award No. DMR-1305119 (Program Manager: Dr. D. Farkas and Dr. D. W. Hess).

\section{Compliance with ethical standards}

Conflicts of interest The authors declare that they have no conflicts of interest.

\section{References}

1. Bolton AJ (1994) Natural fibers for plastic reinforcement. Mater Technol 9:12-20. https://doi.org/10.1080/10667857.1994.11785 012

2. Sanadia R, Caulfield DF, Rowell RM (1994) Additives—reinforcing polypropylene with natural fibers. Plast Eng 50:27-28

3. Chen H-L, Porter RS (1994) Composite of polyethylene and kenaf, a natural cellulose fiber. J Appl Polym Sci 54:1781-1783

4. Hornsby PR, Hinrichsen E, Tarverdi K (1997) Preparation and properties of polypropylene composites reinforced with wheat and flax straw fibres: Part II analysis of composite microstructure and mechanical properties. J Mater Sci 32:1009-1015. https:// doi.org/10.1023/A:1018578322498

5. Thornton K, Poulsen HF (2008) Three-dimensional materials science: an intersection of three-dimensional reconstructions and simulations. MRS Bull 33:587-595. https://doi.org/10.1557/ mrs2008.123

6. Kinney JH, Nichols MC (1992) X-Ray tomographic microscopy (XTM) using synchrotron radiation. Annu Rev Mater Sci 22:121152. https://doi.org/10.1146/annurev.ms.22.080192.001005

7. Baruchel J, Bleuet P, Bravin A et al (2008) Advances in synchrotron hard $\mathrm{x}$-ray based imaging. Comptes Rendus Phys 9:624-641

8. Kaira CS, Stannard TJ, De Andrade V, De Carlo F, Chawla N (2019) Novel deformation mechanisms in aluminum-copper alloys using in situ 4D nanomechanical testing. Acta Mater 176:242-249

9. Hruby P, Singh SS, Williams JJ et al (2014) Fatigue crack growth in $\mathrm{SiC}$ particle reinforced $\mathrm{Al}$ alloy matrix composites at high and low R-ratios by in situ X-ray synchrotron tomography. Int J Fatigue 68:136-143. https://doi.org/10.1016/j.ijfat igue.2014.05.010

10. Singh S, Kaira CS, Bale H et al (2019) In situ micropillar compression of $\mathrm{Al} / \mathrm{SiC}$ nanolaminates using laboratory-based nanoscale
X-ray microscopy: effect of nanopores on mechanical behavior. Mater Charact 150:207-212. https://doi.org/10.1016/j.match ar.2019.02.030

11. Mertens JCE, Henderson K, Cordes NL et al (2017) Analysis of thermal history effects on mechanical anisotropy of 3D-printed polymer matrix composites via in situ x-ray tomography. J Mater Sci 52:12185-12206. https://doi.org/10.1007/s1085 3-017-1339-4

12. Withers PJ (2007) X-ray nanotomography. Mater Today 10:26-34. https://doi.org/10.1016/S1369-7021(07)70305-X

13. Patterson BM, Cordes NL, Henderson K et al (2016) In situ laboratory-based transmission x-ray microscopy and tomography of material deformation at the nanoscale. Exp Mech 56:1585-1597. https://doi.org/10.1007/s11340-016-0197-3

14. Kawasumi M, Hasegawa N, Kato M et al (1997) Preparation and mechanical properties of polypropylene-clay hybrids. Macromolecules 30:6333-6338. https://doi.org/10.1021/ma961786h

15. Zhou Y, He J, Hu J et al (2015) Evaluation of polypropylene/polyolefin elastomer blends for potential recyclable HVDC cable insulation applications. IEEE Trans Dielectr Electr Insul 22:673681. https://doi.org/10.1109/TDEl.2015.7076762

16. Karian H (2003) Handbook of polypropylene and polypropylene composites. CRC Press, Boca Raton

17. Williams JJ, Flom Z, Amell AA et al (2010) Damage evolution in $\mathrm{SiC}$ particle reinforced $\mathrm{Al}$ alloy matrix composites by x-ray synchrotron tomography. Acta Mater 58:6194-6205. https://doi. org/10.1016/j.actamat.2010.07.039

18. Wang Y, De Carlo F, Mancini DC et al (2001) A high-throughput $x$-ray microtomography system at the advanced photon source. Rev Sci Instrum 72:2062-2068. https://doi.org/10.1063/1.13552 70

19. Dhal BB, Peele AG, McMahon PJ et al (2006) Bending magnet source: a radiation source for $\mathrm{X}$-ray phase contrast tomography. Radiat Phys Chem 75:2004-2007. https://doi.org/10.1016/j. radphyschem.2005.10.038

20. Gürsoy D, De Carlo F, Xiao X, Jacobsen C (2014) TomoPy: a framework for the analysis of synchrotron tomographic data. J Synchrotron Radiat 21:1188-1193. https://doi.org/10.1107/S1600 577514013939

21. Münch B, Trtik P, Marone F, Stampanoni M (2009) Stripe and ring artifact removal with combined wavelet-Fourier filtering. Opt Exp 17:8567-8591. https://doi.org/10.1364/OE.17.008567

22. Paganin D, Mayo SC, Gureyev TE et al (2002) Simultaneous phase and amplitude extraction from a single defocused image of a homogeneous object. J Microsc 206:33-40. https://doi.org/10. 1046/j.1365-2818.2002.01010.x

23. Miqueles EX, Rinkel J, O'Dowd F, Bermúdez JSV (2014) Generalized Titarenko's algorithm for ring artefacts reduction. J Synchrotron Radiat 21:1333-1346. https://doi.org/10.1107/S160057751 4016919

24. Kak AC, Slaney M (1999) Algebraic reconstruction algorithms. Principles of computerized tomographic imaging. SIAM, Philadelphia, pp 275-296

25. Fischer-Cripps AC (2004) Nanoindentation. Springer, New York

26. Singh SS, Schwartzstein C, Williams JJ et al (2014) 3D microstructural characterization and mechanical properties of constituent particles in Al 7075 alloys using x-ray synchrotron tomography and nanoindentation. J Alloys Compd 602:163-174. https://doi. org/10.1016/j.jallcom.2014.03.010

27. Handbook for the Aramis system by GOM Gmbh, Gesellschaft fur Optische Mebtechnik, Version 2006, Braunschweig, Germany

28. Sachs C, Fabritius H, Raabe D (2006) Experimental investigation of the elastic-plastic deformation of mineralized lobster cuticle by digital image correlation. J Struct Biol 155:409-425. https:// doi.org/10.1016/j.jsb.2006.06.004 
29. Raabe D, Sachtleber M, Zhao Z et al (2001) Micromechanical and macromechanical effects in grain scale polycrystal plasticity experimentation and simulation. Acta Mater 49:3433-3441. https://doi.org/10.1016/S1359-6454(01)00242-7

30. Kuo JC, Zaefferer S, Zhao Z et al (2003) Deformation behavior of aluminum bicrystals. Adv Eng Mater 5:563-566. https://doi. org/10.1002/adem.200300372

31. Sachtleber M, Zhao Z, Raabe D (2002) Experimental investigation of plastic grain interaction. Mater Sci Eng A 336:81-87. https://doi.org/10.1016/S0921-5093(01)01974-8

32. Roux S, Hild F (2006) Stress intensity factor measurements from digital image correlation: post-processing and integrated approaches. Int J Fract 140:141-157

33. Limodin N, Réthoré J, Buffière JY et al (2009) Crack closure and stress intensity factor measurements in nodular graphite cast iron using three-dimensional correlation of laboratory $x$-ray microtomography images. Acta Mater 57:4090-4101. https:// doi.org/10.1016/j.actamat.2009.05.005

34. Kuo JC, Zaefferer S, Zhao Z et al (2003) Deformation behavior of aluminum bicrystals. Adv Eng Mater 5:563-566. https://doi. org/10.1002/adem.200300372

35. Perona P, Malik J (1990) Scale-space and edge detection using anisotropic diffusion. IEEE Trans Pattern Anal Mach Intell 12:629639. https://doi.org/10.1109/34.56205

36. Perona P, Malik J (1988) A network for multiscale image segmentation. IEEE Int Symp Circuits Syst 1988:2565-2568. https://doi. org/10.1109/ISCAS.1988.15465
37. Duda R, Hart P, Stork D (1995) Pattern classification and scene analysis, 2nd edn. Wiley, New York. https://doi. org/10.2307/1573081

38. Vincent L, Vincent L, Soille P (1991) Watersheds in digital spaces: an efficient algorithm based on immersion simulations. IEEE Trans Pattern Anal Mach Intell 13:583-598. https:// doi.org/10.1109/34.87344

39. Torquato S (1997) Effective stiffness tensor of composite media-I. Exact series expansions. J Mech Phys Solids 45:14211448. https://doi.org/10.1016/S0022-5096(97)00019-7

40. Torquato S (2012) Random heterogeneous materials microstructure and macroscopic properties. Springer, New York

41. Hlushkou D, Liasneuski H, Tallarek U, Torquato S (2015) Effective diffusion coefficients in random packings of polydisperse hard spheres from two-point and three-point correlation functions. J Appl Phys 118:124901. https://doi.org/10.1063/1.4931153

42. Hashin Z, Shtrikman S (1962) A variational approach to the theory of the elastic behaviour of polycrystals. J Mech Phys Solids 10:343-352. https://doi.org/10.1016/0022-5096(62)90005-4

Publisher's Note Springer Nature remains neutral with regard to jurisdictional claims in published maps and institutional affiliations. 